Ключевые слова:

радионуклидная терапия,

паллиативное лечение,

метастатическое поражение

костей

Keywords:

radionuclide therapy,

palliative treatment,

metastatic bones disease.

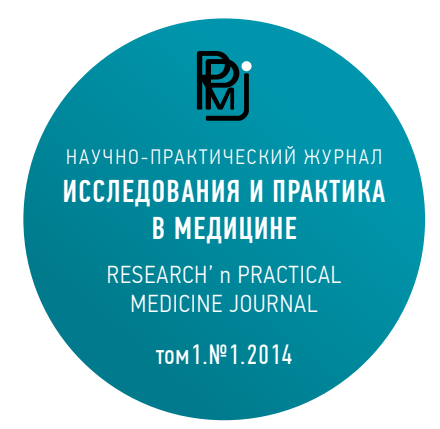

\section{ВОЗМОЖНОСТИ СИСТЕМНОЙ РАДИОНУКЛИДНОЙ ТЕРАПИИ В ПАЛЛИАТИВНОМ ЛЕЧЕНИИ БОЛЬНЫХ С МЕТАСТАТИЧЕСКИМ ПОРАЖЕНИЕМ КОСТЕЙ}

Каприн А. Д.', Костин А. А. ${ }^{1}$, Леонтьев А. В. ${ }^{2}$, Лазутина Т. Н. ${ }^{2}$, Пылова И. В. ${ }^{2}$, Душакова Л.В. ${ }^{2}$

'ФГБУ «ФМИЦ им. П. А. Герцена» Минздрава России (Обнинск, Российская Федерация) 249036, Российская Федерация, Калужская область, г. Обнинск, ул. Королёва, д. 4

${ }^{2}$ МНИОИ им. П.А.Герцена — филиал ФГБУ «ФМИЦ им. П.А.Герцена» Минздрава России (Москва, Российская Федерация)

125284, Российская Федерация, Москва, 2-ой Боткинский проезд, д. 3.

\section{Резюме:}

Болевой синдром, сопровождающий метастатическое поражение костей, существенно снижает качество жизни больных. В последние десятилетия в медицинскую практику внедрены методы системной радионуклидной терапии метастазов в кости с использованием различных радионуклидов, что позволяет не только купировать болевой синдром, но и приостанавливать прогрессирование заболевания. Результаты их применения дают основание считать перспективным развитие в ядерной медицине направления по лечению костных метастатических поражений с помощью системной радионуклидной терапии.

\section{POSSIBILITIES OF SYSTEMIC RADIONUCLIDE THERAPY IN PALLIATIVE TREATMENT OF PATIENTS WITH METASTATIC BONES DISEASE}

Kaprin A.D. ${ }^{1}$, Kostin A.A. ${ }^{1}$, Leontyev A.V. ${ }^{2}$, Lazutina T.N. ${ }^{2}$, Pylova I.V. ${ }^{2}$, Dushakova L.V. ${ }^{2}$

${ }^{1}$ Hertsen FMRC MH RF (Obninsk, Russian Federation)

4, st. Korolev, 249036, Russian Federation, Kaluga region, Obninsk

2 Moscow Oncology Institute of the Hertsen FMRC MH RF (Moscow, Russian Federation)

3, 2 Botkinskiy proezd, 125284, Russian Federation, Moscow

\section{Abstract:}

The pain syndrome accompanying metastatic damage of bones, significantly reduces quality of patients life. In recent decades, medical practice implemented methods systemic radionuclide therapy of bone metastases using different radionuclides, which allows to stop both the pain and the progression of the disease. Results of their application give the grounds to consider the perspective development in nuclear medicine of the direction on treatment bone metastatic defeats by means of systemic radionuclide therapy.

\section{Для корреспонденции:}

Пеонтьев Алексей Викторович,

кандидат медицинских наук, заведующий отделением радионуклидной диагностики МНИОИ им. П.А.Герцена — филиал ФГБУ «ФМИЦ им. П.А.Герцена» Минздрава России Адрес: 125284 , Российская Федерация, Москва,

2-ой Боткинский проезд, д. 3.

Телефон: +7 (495) 945-87-18

e-mail: aleksleont@yandex.ru

Статья поступила 27.10.2014,

принята к печати 12.12.2014

For correspondence:

Leontiev Alexey Victorovich, PhD, head of nuclear medicine Moscow Oncology Institute

of the Hertsen FMRC MH RF

3, 2 Botkinskiy proezd, 125284 ,

Russian Federation, Moscow

Tel: +7 (495) 945-87-18,

e-mail: aleksleont@yandex.ru
Лечение болевого синдрома, обусловленного метастатическим поражением костей, является одной из актуальных проблем оказания паллиативной помощи онкологическим больным. Частота костно-метастатических форм рака молочной железы, предстательной железы, легкого, почек, щитовидной

Сильные боли в костях, ограничение подвижности, патологические переломы, компрессия спинного мозга - основные факторы, снижающие качество жизни пациентов с метастатическим поражением костей. Учитывая относительно длительную выживаемость в этой группе больных, необходимо проведение эффективного паллиативного лечения для улучшения качества жизни пациентов.

В последние десятилетия возрос интерес к радионуклидной терапии множественного метастатического поражения костей скелета при опухолях железы и других органов составляет в среднем по России 65\% [1]. 
различных локализаций [2,3]. Метод основан на способности некоторых $\beta$-излучающих радиофармпрепаратов накапливаться в костных метастазах или пограничных зонах между опухолью и костью. Воздействие $\beta$-излучения радионуклидов на опухолевые клетки приводит к их гибели, снижению митотической активности, редукции опухолевой инфильтрации, вследствие чего замедляется прогрессирование костных метастазов, в ряде случаев отмечается регрессия.

Радиофармпрепараты, применяемые для лечения больных с костными метастазами, должны удовлетворять таким требованиям, как: высокое и селективное накопление в метастатических очагах, быстрое выведение из здоровых тканей, максимальная энергия $\beta$-излучения от 0,8 до 2,0 МэВ, длина максимального пробега $\beta$-частиц в тканях не более 1 см, не должно быть обязательной необходимость в госпитализации пациента, оптимальное соотношение цена/качество, простота производства, удобство доставки и стабильность поставок.

Множественное метастатическое поражение костей, выраженный болевой синдром, прогрессирование метастатического поражения костей на фоне предыдущего лечения, в частности при развитии гормонорезистентности - являются показаниями для проведения системной радионуклидной терапии. Необходимое условие для радионуклидной терапии - позитивный результат остеосцинтиграфии (активное накопление в метастазах диагностических остеотропных препаратов-Тс ${ }^{99 m}$ ).

В мировой практике для паллиативной терапии костных метастазов используются радиофармпрепараты

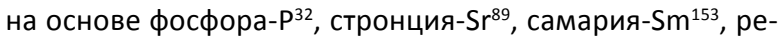

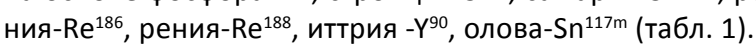

${ }^{32} \mathrm{P}$ - двузамещенный фосфат натрия - чистый $\beta$-излучатель с периодом полураспада 14,3 суток. Отсутствие в спектре излучения $ү$-лучей не позволяет проводить обследование пациентов на однофотонных эмиссионных компьютерных томографах, контролировать распределение радиофармпрепарата в организме. Максимальная энергия $\beta$-частиц - 1,71 МэВ, средняя - 0,695 МэВ. Препарат вводят перорально, разовая активность составляет 200-225 МБк, на курс лечения 400-450 МБк. Недостатком этого радиофармпрепарата является значительная миелотоксичность, которая проявляется лейкопенией, тромбоцитопенией или панцитопенией. Панцитопения проявляется через 4-5 недель с возобновлением кроветворения на 6-7 неделе и является дозозависимой. Предпринимались попытки совместного применения ${ }^{32} \mathrm{P}$ с тестостероном, стимулирующим костный мозг, при этом у 87\% пациентов отмечен аналгезирующий эффект, но сохранялась выраженная миелотоксичность [4].
Оптимальными клиническими и физическими параметрами обладает стронция-Sr ${ }^{89}$ хлорид. ${ }^{89} \mathrm{Sr}$ $\beta$-излучатель с периодом полураспада 50,5 суток является химическим аналогом кальция, что обеспечивает его избирательное накопление в очагах с повышенной остеобластической активностью. Максимальная энергия $\beta$-излучения - 1,46 МэВ, средняя - 0,583 МэВ. Пробег $\beta$-частиц в мягких тканях - 8 мм, в костях - 3,5 мм. Накопление препарата в костной ткани пропорционально объему метастатического поражения и составляет от 20 до 80\% введенной активности. ${ }^{89} \mathrm{Sr}$-хлорид остается в очагах поражения около 100 суток, непораженная метастазами костная ткань накапливает незначительную часть введенной активности и теряет ее в течение 14 суток. Соотношение доз метастаз/костный мозг составляет 10:1. Препарат вводят внутривенно - 150 МБк (из расчета 2 МБк/кг массы тела). Возможно амбулаторное применение ${ }^{89} \mathrm{Sr}$-хлорида.

Аналгезирующий эффект наступает через 7-20 суток после инъекции препарата, продолжается в течение 4-6 месяцев после однократной инъекции, снижение интенсивности болевого синдрома отмечают более чем у $80 \%$ пациентов. Повторное введение препарата возможно через 4-6 месяцев (оптимальная лечебная тактика - введение препарата 2 раза в год). Многократное применение ${ }^{89} \mathrm{Sr}$-хлорида приводит к миелосупрессии.

В клинической практике используются два препарата стронция: стронция - $\mathrm{Sr}^{89}$ хлорид, производства «Медрадиопрепарат» и препарат Метастрон, производства фирмы «Амершам».

Российские и зарубежные авторы $[5,6]$ свидетельствуют о достаточно высокой эффективности $\mathrm{Sr}^{89}$-хлорид у больных с метастазами опухолей различных локализаций. Сообщается о противоболевом эффекте в 47-100\% случаев. При этом наиболее выраженный эффект отмечен у больных с метастазами рака молочной и предстательной железы.

Для повышения эффективности лечения предложено сочетанное лучевое воздействие, включающее на первом этапе локальное дистанционное облучение очагов наиболее выраженной болевой активности с последующим введением ${ }^{89} \mathrm{Sr}$-хлорида. Поводом для этого послужили сведения об усилении остеобластической активности в ранней фазе репарации костной ткани в ответ на успешную терапию костных метастазов. В качестве подобной терапии используют дистанционное локальное облучение. Усиление репаративной активности способствует более интенсивному поглощению стронция- 89 в очаге метастатического поражения. Сочетанная лучевая терапия, включающая системную терапию препаратом хлорид стронция- ${ }^{89}$ и локальную лучевую терапию,

Таблица 1

\begin{tabular}{l|l|l|lccc}
\hline Нуклид & Sr-89 & Sm-153 & Re-186 & Re-188 & P-32 & Y-90 \\
\hline Период полураспада (дни) & 52 & 2,1 & 3,8 & 0,7 & 14 & 2,7 \\
\hline Энергия бета-излучения (МэB) & 1,4 & 0,8 & 1,1 & 2,1 & 1,7 & 2,27 \\
\hline Максимальный пробег в мягких тканях (мм) & 6,6 & 3,7 & 4,6 & 10,0 & 8,1 & 10,9 \\
\hline
\end{tabular}


позволяет повысить противоболевой эффект на 10\%, в том числе у больных с тяжелым болевым синдромом на 30\%, и увеличить на 3 месяца его продолжительность по сравнению с локальной лучевой терапией. Сочетанная лучевая терапия является методом выбора при лечении больных с костными метастазами рака предстательной и молочной железы [7].

Наряду со стронцием ${ }^{89} \mathrm{Sr}$-хлоридом в России используются самарий-оксабифор $\left({ }^{153} \mathrm{Sm}\right)$, который является первым отечественным остеотропным радиотерапевтическим препаратом (рег. № 000008/01-2000, патент РФ № 2162714 от 20.06.2000). По механизму действия близок к зарубежному аналогу ${ }^{153} \mathrm{Sm}$-EDTMP фирмы («CIS Bio International»).

Самарий-оксабифор $\left({ }^{153} \mathrm{Sm}\right)$ - смешанный $(\beta-, \gamma-)$ излучатель с средней энергией $\beta$-частиц 233 КэВ и гамма-квантов 103 КэВ (выход 29\%). Период полураспада ${ }^{153} \mathrm{Sm}$ - 46,3 часа. Средний пробег $\beta$-частиц в костной ткани - 1,7 мм, в мягких тканях - 3,1 мм. После внутривенного введения препарат накапливается в костной ткани, преимущественно в метастазах. Очаги накопления хорошо визуализируются при исследовании на гамма-камере, что делает возможным проводить сцинтиграфический контроль в процессе лечения. Терапия самарием-оксабифором $\left({ }^{153} \mathrm{Sm}\right)$ позволяет достигнуть стойкого подавления болевого синдрома. Клинически значимая эффективность (полный + выраженный + частичный эффект) при раке молочной железы через 1 , 3 и 6 мес. 80,5\% 76,5\%, 64,1\%, соответственно, при раке предстательной железы - 81,7\%, 76,9\%, и 61,8\%. Динамика средних показателей интенсивности болей при раке молочной железы - 5,9 балла (до лечения), 3,6 (через 1 месяц), 4,0 (через 3 месяца) и 4,7 (через 6 месяцев), при раке предстательной железы - 6,1 балла (до лечения), 3,8, 4,3 и 4,9 баллов (через 1, 3 и 6 месяцев соответственно) [8]. Препарат неэффективен у больных раком легкого. В США ${ }^{153} \mathrm{Sm}-\mathrm{EDTMP}$ в настоящее время является одним из наиболее распространенных радиофармпрепаратов применяемых для паллиативного лечения костных метастазов.

Кроме того, продолжается изучение новых перспективных радиофармпрепаратов для радионуклидной терапии метастазов в скелете.

${ }^{186} \mathrm{Re}-\mathrm{HEDP}$ ( ${ }^{186}$ рений-гидроксиэтилендифосфонат) смешанный ( $\left.\beta-, \gamma^{-}\right)$излучатель с максимальной энергией $\beta$-частиц 1,07 МэВ, средней - 0,349 МэВ и ү-квантов 0,137 МэВ. Средняя длина пробега в мягких тканях 1,1 мм. Период полураспада радионуклида - 88,9 часов. Радиофармпрепарат вводят внутривенно активностью 1300 МБк. Дозолимитирующей токсичностью является тромбоцитопения. Средний срок восстановления костного мозга составляет от 4 до 6 недель. Соотношение между накоплением РФП в опухоли и костном мозге 14:1. Через сутки после введения 34,2-66,3\% препарата накапливается в костях. Противоболевой эффект составляет 70-80\% при средней продолжительности эффекта 4 недели $[9,10]$. Малая токсичность рения позволяет вводить этот изотоп повторно.

Представляет интерес использование генераторного радионуклида ${ }^{188} \operatorname{Re}-\mathrm{HEDP}$, который обладает выра- женным сродством к остеокластам, период полураспада 16,7 часов. Достоинством этого генератора является наличие как высокоэнергетического $\beta$-излучения (максимальная энергия - 2,12 МэВ, средняя - 0,78 МэВ), так и гамма-составляющей с энергией 0,155 МэВ. Биораспределение и дозиметрические характеристики ${ }^{188} \mathrm{Re}$ HEDP идентичны ${ }^{186} \mathrm{Re}-\mathrm{HEDP}$, что обусловливает аналогичные клинические эффекты и проявления токсичности при лечении метастазов. Зарубежные авторы [11] сообщают о высокой эффективности применения ${ }^{186} \mathrm{Re}-\mathrm{HEDP}$ у больных с метастазами опухолей различных локализаций. Терапия ${ }^{186} \mathrm{Re}-\mathrm{HEDP}$ позволяет достичь существенное облегчение боли в костях у $80 \%$ пациентов, у 77\% больных раком легких, у 80\% - раком предстательной железы, у 83\% - раком молочной железы, у 100\% - раком мочевого пузыря, у 50\% - раком почки, у 50\% - раком носоглотки. Согласно данным Palmedo Н. (2003), после двукратного введения ${ }^{186} \mathrm{Re}-\mathrm{HEDP}$ с интервалом в 8 недель у пациентов с метастатическим гормонорезистентным раком предстательной железы отмечено уменьшение выраженности болевого синдрома в 92\% случаев, а снижение уровня ПСА>50\% - в 39\% случаев [12]. Серьезных побочных эффектов или миелотоксичности не отмечено.

В России завершены доклинические исследования по применению для паллиативной терапии костных метастазов радиофармацевтического препарата, представляющего собой комплекс рения- ${ }^{188}$ с золедроновой кислотой [13]. Полученные результаты дают основание говорить о перспективности применения РФП ${ }^{188} \mathrm{Re}$-золедроновая кислота для терапии патологии, сопровождающейся ремоделированием костной ткани, в том числе метастатических поражений скелета.

${ }^{117} \mathrm{mSn}$-DTPA излучает электроны внутренней конверсии с коротким пробегом в костной ткани. Период полураспада изотопа - 14,03 суток. Энергия электронов внутренней конверсии - 0,129 и 0,153 МэВ, средняя длина их пробега составляет 0,2-0,3 мм, что обусловливает низкую частоту миелосупрессии при его применении. Энергия ү-излучения - 0,159 МэВ. РФП вводят внутривенно активностью 370-740 МБк. ${ }^{117} \mathrm{mSn}$-DTPA не имеет аффинности к гидроксиапатиту, накапливается путем преципитации оксида олова на поверхности кости или за счет гидролитической реакции с гидроксиапатитом. у 75\% пациентов отмечают аналгезирующий эффект, он проявляется через неделю после начала терапии. Наибольший опыт применения этого препарата в США.

${ }^{177}$ Lu-EDTMP излучает относительно мягкое 及-излучение (максимальная энергия - 0,497 МэВ), электроны внутренней конверсии с энергиями 0,133 и 0,014 МэВ и ү-излучение с энергией 0,113 МэВ. Период полураспада изотопа - 6,71 суток. Эти свойства позволяют достигать хорошего терапевтического паллиативного эффекта в сочетании с относительно низкой миелотоксичностью, что особенно важно при повторном введении препарата.

Наличие в спектре излучения радионуклидов ${ }^{153} \mathrm{Sm}$, ${ }^{186} \mathrm{Re},{ }^{188} \mathrm{Re},{ }^{117} \mathrm{mSn},{ }^{177} \mathrm{Lu} \mathrm{\gamma}$-лучей позволяет проводить обследование пациентов на однофотонных эмиссионных компьютерных томографах, контролировать распределение РФП в организме. 
Препарат «Ксофиго» (Радий 223 -дихлорид) является совместной разработкой фирм «Bayer» и «Algeta», перспективен для лечения костных метастазов при кастрационно-резистентном раке предстательной железы. Радий 223 - альфа-излучатель. По результатам III фазы клинических испытаний (921 пациент, страдающий кастрационно-резистентным раком предстательной железы) препарат «Ксофиго» способствует увеличению общей средней выживаемости до 14,9 месяцев по сравнению с 11,3 месяцами в группе плацебо, также среди пациентов, получавших «Ксофиго», зафиксировано значительно меньшее количество серьезных побочных эффектов - 47\% (в контрольной группе этот показатель составил 60\%) [14].

\section{Информация о соавторах:}

1. Каприн Андрей Дмитриевич, член-корр РАН, доктор медицинских наук, профессор, заведующий кафедрой урологии и оперативной нефрологии с курсом онкоурологии медицинского факультета Медицинского института РУДН, генеральный директор ФГБУ «Федерального медицинского исследовательского центра имени П.А.Герцена» Минздрава России, Заслуженный врач России. ORCID 0000-0001-8784-8415

2. Костин Андрей Александрович, доктор медицинских наук, профессор, заведующий кафедрой урологии, онкологии, нефрологии ФПК МР Медицинского института РУДН, Первый заместитель генерального директора ФГБУ «Федерального медицинского исследовательского центра имени П.А.Герцена» Минздрава России. ORCID 0000-0002-0792-6012

3. Лазутина Татьяна Николаевна, кандидат медицинских наук, врач-радиолог отделения радионуклидной диагностики ФГБУ «МНИОИ им. П.А. Герцена» - филиал ФГБУ «ФМИЦ им. П.А.Герцена» Минздрава России .

4. Пылова Ирина Валентиновна, кандидат медицинских наук, врач-радиолог отделения радионуклидной диагностики ФГБУ «МНИОИ им. П.А. Герцена» - филиал ФГБУ «ФМИЦ им. П.А.Герцена» Минздрава России.

5. Душакова Любовь Владимировна, документовед отделения диагностики и лечения ФГБУ “ФМИЦ им. П.А. Герцена” Минздарава России и по совместительству - заведующая лабораторией кафедры Урологии и оперативной нефрологии с курсом онкоурологии медицинского факультета Медицинского института РУДН.
Таким образом, радионуклидная терапия с успехом применяется у больных с метастазами в кости различных злокачественных опухолей, позволяя уменьшить болевой синдром, затормозить его дальнейшее прогрессирование, снизить потребность в анальгетиках, а главное - повысить качество жизни пациентов. Наиболее эффективна радионуклидная терапия в паллиативном лечении больных раком предстательной и молочной железы. Учитывая успешный мировой опыт применения радионуклидной терапии у больных с метастазами в кости, следует развивать данный метод в практике отечественного здравоохранения.

Information about co-authors:

1. Kaprin Andrei Dmitrievich, RAS Corr. Mem., MD, Prof.; Corresponding Member of the Russian Academy of Education;Honored Physician of the Russian Federation; General Director Hertsen Federal Medical Research Centr of the Ministry of Health of the Russian Federation; Director Moscow Oncology Institute of the Hertsen FMRC MH RF, Head, Department of Urology with Course of Urological Oncology, Faculty for Postgraduate Training, Peoples' Friendship University of Russia; Principal Urologist, Academy of Sciences of the Russian Federation ORCID 0000-0001-8784-8415

2. Kostin Andrei Aleksandrovich, MD., Prof., First Deputy General Director Hertsen Federal Medical Research Centr of the Ministry of Health of the Russian Federation ORCID 0000-0002-0792-6012

3. Lazutina Tatiana Nikolaevna, MD, radiologist department of nuclear medicine Moscow Oncology Institute of the Hertsen FMRC MH RF

4. Pylova Irina Valentinovna, MD, radiologist separating radionuclide diagnostics Moscow Oncology Institute of the Hertsen FMRC MH RF

5. Dushakova Lybov Vladimirovna, separation, document the diagnosis and treatment Moscow Oncology Institute of the Hertsen FMRC MH RF, Head of the Laboratory of the Department of Urology and Surgical Nephrology oncourology with the course of the Medical Faculty of the Medical Institute of People's Friendship University. 
1. Меньшикова Е.С. Паллиативное лечение больных с костными метастазами злокачественных опухолей с использованием стронция-89 хлорида // Автореф. к.м.н. - 2008 г.

2. Riccabona G. Nuclear medicine in diagnosis and therapy of bone and joint diseases [Journal] // Nucl. Med. Rev. - 1999. Vol. 2. - pp. 42-52.

3. Kraeber-Bodere F., Campion L., Rousseau C. et al. Treatment of bone metastases of prostate cancer with strontium-89 chloride: efficacy in relation to the degree of bone involvement [Journal] // Eur. J. Nucl. Med. - 2000. - Vol. 27. - pp. 1487-1493.

4. Maxfield J.J.R., Maxfield J.G.S., Maxfield W.S. The use of radioactive phosphorous and testosterone on metastatic bone lesions from breast and prostate [Journal] // Sth. Med. J. - 1958. - Vol. 35. - p. 320.

5. Berna L., Carrio I., Alonso C. Bone pain palliation with strontium -89 in breast cancer patiens with bone metastases and refractory bone pain [Journal] // Eur. J. Nucl. Med. - 1995. - Vol. 22. - pp. 1101-1104.

6. Васильев А.Ю., Щербаков С.Д., Краснова Э.В. и др. Опыт лечения костных метастазов хлоридом стронция-89 [Журнал] // Мед. радиология и радиац. безопасность. - 2003 г. Т. 48. - стр. 66-69.

7. Меньшикова Е.С., Важенин А.В., Шарабура Т.М., Ваганов Н.В. Оценка эффективности стронция-89 хлорида в сочетании с дистанционной лучевой терапией костных метастазов рака предстательной и молочной желез [Журнал] // Сибирский онкологический журнал. - 2008 г. - Т. 3. - стр. 34-37.

\section{References}

1. Menshikova E.S. Palliativnoe lechenie bol'nyh s kostnymi metastazami zlokachestvennyh opuholej $\mathrm{s}$ ispol'zovaniem stroncija-89 hlorida // Avtoref. k.m.n. - 2008 g.

2. Riccabona G. Nuclear medicine in diagnosis and therapy of bone and joint diseases [Journal] // Nucl. Med. Rev. - 1999. Vol. 2. - pp. 42-52.

3. Kraeber-Bodere F., Campion L., Rousseau C. et al. Treatment of bone metastases of prostate cancer with strontium- 89 chloride: efficacy in relation to the degree of bone involvement [Journal] // Eur. J. Nucl. Med. - 2000. - Vol. 27. - pp. 1487-1493.

4. Maxfield J.J.R., Maxfield J.G.S., Maxfield W.S. The use of radioactive phosphorous and testosterone on metastatic bone lesions from breast and prostate [Journal] // Sth. Med. J. - 1958. - Vol. 35. - p. 320.

5. Berna L., Carrio I., Alonso C. Bone pain palliation with strontium -89 in breast cancer patiens with bone metastases and refractory bone pain [Journal] // Eur. J. Nucl. Med. - 1995. - Vol. 22. - pp. 1101-1104.

6. Vasil'ev A.Ju., Shherbakov S.D., Krasnova Je.V. i dr. Opyt lechenija kostnyh metastazov hloridom stroncija-89 [Zhurnal] // Med. radiologija i radiac. bezopasnost'. - 2003 g. - T. 48. - str. 66-69.

7. Menshikova E.S., Vazhenin A.V., Sharabura T.M., Vaganov N.V. Ocenka jeffektivnosti stroncija-89 hlorida $v$ sochetanii $s$ distancionnoj luchevoj terapiej kostnyh metastazov raka predstatel'noj i molochnoj zhelez [Zhurnal] // Sibirskij onkologicheskij zhurnal. - 2008 g. - T. 3. - str. 34-37.
8. Крылов В.В. Радионуклидная терапия самарием оксабифором, $153 \mathrm{Sm}$ при метастатическом поражении костей // Автореферат. - Обнинск : [б.н.], 2007 г.

9. Palmedo H., Bender H., Schomburg A. Pain therapy with rhenium-186 HEDP in multiple bone metastases [Journal] // Nuklearmedizin. - 1996. - Vol. 35. - pp. 63-67.

10. Guerra U.P., Englaro E., Cattruzzi E. Palliative therapy with rhenium-186-HEDP for bone metastases of breast cancer [Journal] // Tumori. - 1997. - Vol. 83. - pp. 560-62.

11. Li S., Liu J., Zhang H. et al. Rhenium-188 HEDP to treat painful bone metastases [Journal] // Clin Nucl Med. - 2001. - Vol. 26. - pp. 919-22.

12. Palmedo H., Manka Waluch A., Albers P. et al. Repeated bone_targeted therapy for hormone_refractory prostate carcinoma:Randomized phase II trial with the new, high_energy radiopharmaceutical rhenium_188 hydroxyethylidenediphosphonate [Journal] // J. Clin. Oncol. - 2003. - Vol. 21. - pp. 2869-2875.

13. Волознев Л.В., Клементьева О.Е., Корсунский В.Н. и др. Экспериментальная оценка функциональной пригодности радиофармацевтического препарата 188re-золедроновая кислота [Журнал] // Молекулярная медицина. - 2013 г. - Т. 2. - стр. 42-45.

14. Kluetz P.G., Pierce W., Maher V.E. et al. Radium Ra 223 dichloride injection: U.S. Food and Drug Administration drug approval summary [Journal] // Clin Cancer Res. - 2014. - Vol. 20. - pp. doi: 10.1158/1078-0432.CCR-13-2665. Epub 2013 Nov 4.

8. Krylov V.V. Radionuklidnaja terapija samariem oksabiforom, 153 Sm pri metastaticheskom porazhenii kostej // Avtoreferat. - Obninsk : [b.n.], 2007 g

9. Palmedo H., Bender H., Schomburg A. Pain therapy with rhenium-186 HEDP in multiple bone metastases [Journal] // Nuklearmedizin. - 1996. - Vol. 35. - pp. 63-67.

10. Guerra U.P., Englaro E., Cattruzzi E. Palliative therapy with rhenium-186-HEDP for bone metastases of breast cancer [Journal] // Tumori. - 1997. - Vol. 83. - pp. 560-62.

11. Li S., Liu J., Zhang H. et al. Rhenium-188 HEDP to treat painful bone metastases [Journal] // Clin Nucl Med. - 2001. - Vol. 26. - pp. 919-22.

12. Palmedo H., Manka Waluch A., Albers P. et al. Repeated bone_targeted therapy for hormone_refractory prostate carcinoma:Randomized phase II trial with the new, high_energy radiopharmaceutical rhenium 188 hydroxyethylidenediphosphonate [Journal] // J. Clin. Oncol. - 2003. - Vol. 21. - pp. 2869-2875.

13. Voloznev L.V., Klement'eva O.E., Korsunskij V.N. i dr. Jeksperimental'naja ocenka funkcional'noj prigodnosti radiofarmacevticheskogo preparata 188re-zoledronovaja kislota [Zhurnal] // Molekuljarnaja medicina. - 2013 g. - T. 2.- str. 42-45.

14. Kluetz P.G., Pierce W., Maher V.E. et al. Radium Ra 223 dichloride injection: U.S. Food and Drug Administration drug approval summary [Journal] // Clin Cancer Res. - 2014. - Vol. 20. - pp. doi: 10.1158/1078-0432.CCR-13-2665. Epub 2013 Nov 4. 